\title{
Role of First-trimester 3D Power Doppler of Placental Blood Flow and 3D Placental Volume in Early Prediction of Preeclampsia
}

\author{
Ameer Abdallah ${ }^{1}$, Mohamed Tawfik ${ }^{1}$, Safaa Mohamed ${ }^{1}$, Mazen Abdel-Rasheed ${ }^{2}$, Sondos \\ Salem $^{2}$, and Eissa Khalifa ${ }^{1}$ \\ ${ }^{1}$ Minia University Faculty of Medicine \\ ${ }^{2}$ National Research Centre
}

April 28, 2020

\begin{abstract}
Objective: First-trimester uterine artery Doppler was declared as a useful tool for predicting preeclampsia. Placental volume and vascular indices were recently used to predict preeclampsia. The study aims to assess the role of the first-trimester $3 \mathrm{D}$ placental volume and power Doppler of placental vascular indices combined with the uterine artery Doppler indices in prediction of preeclampsia. Design: A prospective cohort study Sample: 2019 women with a singleton pregnancy in their first-trimester. Methods: At 11-13+ weeks of gestation, 3D trans-abdominal ultrasound with Doppler scan for all participants then they were followed up until delivery. Main Outcome Measures: 3D placental volume, 3D Power Doppler of placental blood flow and uterine artery Doppler in the first trimester. Results: 163 women developed preeclampsia while 1856 women were normotensive. In the preeclampsia group, the placental volume, placental vascular indices (VI, FI, and VFI) showed a significant decrease, while means uterine PI and RI showed a significant increase. The placental vascular indices (VI, FI, and VFI) showed higher sensitivity while the placental volume and the uterine artery Doppler indices (PI and RI) showed higher specificity for the prediction of preeclampsia. Conclusion: Combined early screening of uterine artery Doppler with placental volume and vascular indices could be beneficial to increase the accuracy of early prediction of preeclampsia. Funding: There is no specific grant from any funding agency. Keywords: 3D-Doppler ultrasonography; placental volume; placental vascular indices; uterine artery Doppler; preeclampsia
\end{abstract}

\section{Background}

Preeclampsia; a pregnancy-specific hypertensive disorder; is defined as new-onset hypertension and proteinuria after $20^{\text {th }}$ weeks of gestation, in a previously normotensive woman (1). It almost affects about $2-8 \%$ of all pregnancies, and it remains a major cause of maternal and perinatal morbidity and mortality worldwide since it accounts for about $14 \%$ of all maternal deaths $(2,3)$. Moreover, fetal growth restriction (FGR) that results in small for gestational age (SGA) neonates $(<10$ th percentile) represents a leading cause of perinatal morbidity and mortality. There is a significant overlap between the two conditions as they have a common prevailing theory of pathogenesis which depends on the defective extravillous and endovascular trophoblastic invasion of the decidual spiral arteries that results in impaired blood flow in the inter-villous space with subsequent abnormal placentation (4). This pathogenic mechanism, underpinning these common obstetric syndromes, imply that placental vascular changes that lead up to preeclampsia and FGR can be identified as early as the first trimester $(5,6)$.

The importance of 3D ultrasound has been increasing in reproduction; starting from effective evaluation of infertility cases, such as estimation of antral follicle count and ovarian volume, up to its valuable role during antenatal care (7). The advent of 3D ultrasound, as well as the power Doppler technology, has enabled the non-invasive study of blood flow and organ volumes in various organs including obstetric patients. Previous 
studies have suggested that the impedance of blood flow in the uterine arteries decreases with advancing gestation in normal pregnancy, and hence reflecting the developmental changes in the spiral arteries with development of low resistance vessels (8). However, these changes are defective in preeclampsia, where measurements of the uterine artery Doppler reflect increased impedance to flow in the second trimester (and more recently in the first trimester) in women who developed preeclampsia (9).

It has been reported that, in normal pregnancy, doubling of the placental volume occurs between the $11^{\text {th }}$ and $14^{\text {th }}$ gestational weeks, in parallel with doubling of the fetal size and gestational sac volume (10). Furthermore, the study of placental bed vascular changes using the 3D power Doppler (3D-PD) indices; vascularization index (VI), flow index (FI) and vascularization-flow index (VFI); have suggested a promising role for the 3D-PD in evaluating of the placenta in the first trimester to predict preeclampsia and FGR (11). Although the results of these studies are promising, ultrasound examination of the placenta or uterine arteries alone in the first trimester is not still universally accepted as a screening tool for preeclampsia and FGR due to possibly inconsistency of the methodologies used and the definitions of abnormal indices (9).

The main objective of the present study was to estimate the performance and efficacy of the first-trimester 3D Power Doppler of placental vascular indices and 3D placental volume combined with the uterine artery Doppler indices, as early predicting tools for preeclampsia.

\section{Patients and Methods}

This prospective cohort study was conducted at El-Minia Maternity University Hospital on 2019 women with a singleton pregnancy in their first trimester during the period between July 2016 and June 2019. The Ethics Committee of Faculty of Medicine at El-Minia University approved the study protocol under registration number 1876-5-m-2018. All women enrolled in this study were regularly followed up in the antenatal clinic in El-Minia Maternity University Hospital and have given written informed consent for participation in the study. Inclusion criteria included singleton pregnancy and accurate dating of pregnancy. Women with multiple pregnancies, fetal anomalies or suspected genetic conditions that may affect fetal growth were excluded from this study.

Voluson S8 ultrasound device (GE Healthcare, Chicago, Illinois, USA) was used for both abdominal sonography and Doppler technique, with the trans-abdominal probe frequency of $7 \mathrm{MHz}$ and the examination time was approximately 20 minutes. At $11-13^{+}$weeks of gestation, 3D trans-abdominal ultrasound scan was performed for confirmation of fetal viability, gestational age and careful exploration for any fetal abnormalities. Where there is a discrepancy between the menstrual age and gestational age calculated by ultrasound, the gestational age was determined by the crown-rump length.

For Doppler studies, a sagittal section of the uterus was obtained, where the cervical canal and internal cervical OS could be recognized. Then, the transducer was smoothly tilted from one side to the other. Color flow mapping was used to identify each ascending uterine artery, along both sides of the cervix and uterus at the level of the internal OS, to measure the pulsatility index (PI) and the resistance index (RI) in both uterine arteries, and to detect the presence of a notch and determine whether it is unilateral or bilateral. When three similar consecutive waveforms were obtained, PI and RI for both uterine arteries were measured, and then the mean PI and RI were calculated. An abnormal value was considered if the mean PI and RI are greater than the 95 th percentile.

To view the entire placenta, the transducer was positioned perpendicular to the placenta. The 3D placental scan was adjusted to an angle of $70^{\circ}$ with a maximum region of interest. The external limits of the placenta were defined by the basal plate and the chorionic plate without the myometrium. The placental volume was then calculated using Virtual Organ Computer-Aided Analysis "VOCAL" (GE Healthcare, Chicago, Illinois, USA), with an angle of rotation of 30deg (Figure 1, 2). The axial plane represented the reference, and the calipers were positioned on either side of the placenta.

After calculation of the placental volume, the 3D-PD histogram was used to determine the vascular indices: a) VI, which refers to the color percentage within the placental volume, and indicates the number of vessels 
within the placenta; b) FI, which refers to the ratio of weighted/total color voxel, and indicates the intensity of the placental blood flow at the time of the 3D sweep; and c) VFI, which is automatically calculated from VI and FI. All measures for a group of 30 women were taken twice by the same examiner and then by another examiner on the same day to assess both intra- and inter-observer variability.

During antenatal care, preeclampsia was diagnosed after 20 weeks of gestation if the blood pressure was higher than 140/90 mmHg on two occasions 4 hours apart and proteinuria of $300 \mathrm{mg}$ in 24 hours. FGR was diagnosed if the estimated weight of the fetus is below the 10th percentile for its gestational age. The decision for delivery was based on any evidence of worsening preeclampsia by clinical assessment (uncontrollable blood pressure, development of severe preeclampsia or development of eclampsia) or laboratory investigations (any worsening of hematological or biochemical parameters) or evidence of fetal compromise (nonreactive nonstress test, low biophysical profile, oligohydramnios, reversed end-diastolic flow in the umbilical artery or dropping middle cerebral artery to umbilical artery Doppler ratio). Clinicians, who assessed the maternal and perinatal outcomes, were blinded to the ultrasound studies results and the ultrasound findings in the first trimester did not result in the addition of any intervention apart from the routine antenatal care.

Statistical analysis: Data were statistically analyzed using the Statistical Package for the Social Science "SPSS", Version 25 for Microsoft Windows (Inc., Chicago, IL, USA). Qualitative variables were presented as frequency and percentage, whereas quantitative data were expressed as mean +- SD. Patients who developed preeclampsia and those who did not were compared for continuous and categorical data using t-test and Chisquare respectively. $\mathrm{P}$-value $<0.05$ was considered significant, and that of $<0.001$ as highly significant. ROC curve was used to assess the sensitivity and specificity of the obtained cut-off value. Logistic regression analysis was used to calculate the relative weight and importance of several predictors and to assess the best prediction model. The likelihood ratios were used to calculate the performance of the ultrasound parameters in the prediction of the outcomes.

\section{Results}

The present study included 2257 women; 126 of them were unfulfilling the inclusion criteria, 34 were missing during follow-up and 78 miscarried. Therefore, the total number of women enrolled in this study was 2019. The mean age of all studied cases was $24.55+-5.78$ years, and the mean BMI was 25.15+-2.27. Regarding parity, 987 women (48.9\%) were nulliparous while 1032 women $(51.1 \%)$ were multiparous. The number of cases who developed preeclampsia was 163 cases (8.1\%) while the rest of the study group, 1856 women $(91.9 \%)$, were normotensive.

The results of the present study revealed no statistically significant difference between the preeclampsia and normotensive groups regarding age and BMI. Nulliparous women, previous history and family history of preeclampsia showed a significant increase in the preeclampsia group. Regarding Doppler parameters of the preeclampsia group, placental vascular indices (VI, FI, and VFI) showed a significant decrease, while means uterine PI and RI showed a significant increase. The placental volume has significantly decreased in the preeclampsia group. The incidence of development of FGR was $41.9 \%$ in the preeclampsia group, while it was only $13.3 \%$ in the normotensive groups. Only three cases from the preeclampsia group developed intrauterine fetal death (IUFD). Regarding the neonatal outcome in the preeclampsia group, the neonatal birth weight was significantly decreased but with a significant increase in the incidence of neonatal complications and the rate of NICU admission (Table 1).

ROC curve analysis of the placental vascular indices and uterine artery indices (Figures 3) was used to assess the sensitivity and specificity of the obtained cut-off value of these indices for prediction of preeclampsia (Table 2) . Interestingly, the placental vascular indices (VI, FI, and VFI) showed higher sensitivity while the placental volume and the uterine artery Doppler indices (PI and RI) showed higher specificity for the prediction of preeclampsia. The logistic regression model for the prediction of preeclampsia using the clinical and 3D ultrasound criteria for uteroplacental blood flow was presented in Table 3 . In the multivariate regression model for prediction of preeclampsia, placental volume, placental VI, mean uterine PI, past history and family history of preeclampsia showed significant odd ratio (p-value $<0.05$ ). 


\section{Discussion}

The pathogenesis of most pregnancy complications could be due to placental maldevelopment. Therefore, placental volume, weight, surface area, placental insufficiency, and vascular indices significantly help to evaluate early FGR and gestational complications. Moreover, placental inadequacy which is characterized by insufficient transplacental transport of nutrients and oxygen usually results in FGR (12). Preeclampsia is regarded as the major cause of maternal and perinatal morbidity and mortality. Worldwide, about 76,000 maternal deaths and 500,000 neonatal deaths per year are attributed to preeclampsia (13). Moreover, it has been reported that the clinical pictures of preeclampsia are attributed to various manifestations of organ dysfunction including liver, kidneys, heart, brain, lung, and pancreas (14).

The results of the present study revealed that only 163 cases (8.1\%) developed preeclampsia, 97 of them (59.5\%) were nulliparous while 66 women (40.5\%) were multiparous Luo et al., (2007) did a meta-analysis of 26 studies and reported that nulliparous women had a higher risk of preeclampsia ( 2.42 fold) than multiparous patients. This may be attributed to some factors including angiogenic profile, differences in immune response or insulin resistance reactivity pattern between primiparous and multiparous women. In another metaanalysis, Wang et al., (2013) concluded that overweight/obese women showed higher preeclampsia risk (2-4 folds) compared to those with normal weight.

Consistent with our results, Yucesoy et al., (2005) and Musa et al., (2018) have reported that positive history of preeclampsia may affect its incidence which confirms that this positive history is a significant risk factor for its occurrence. They also found that obesity is a risk factor for preeclampsia. In our study, although the preeclampsia group showed a higher BMI than the normotensive group yet, this difference was insignificant as most participants in our study were overweight representing most women in Egypt $(15,16)$.

Regarding the uterine artery indices, uterine artery Doppler is regarded as promising potential screening tool for the prediction of early-onset preeclampsia in the first trimester (17). Moreover, it has been used to identify the "at risk" population in the first trimester $(18,19)$.

The present study revealed that preeclamptic cases had significantly higher PI and RI than the normal cases (p[?] 0.001). These results are consistent with those concluded by Khalil et al., (2010) who investigated the predictive value of first-trimester uterine artery PI and RI for assessing the risk of preeclampsia in high-risk women. They found that PI and RI increased significantly in the presence of preeclampsia. They also concluded that the mean value of PI and pulse-wave analysis can help in the prediction of preeclampsia in women at increased risk (20).

Visser et al., (2014) and Schoots et al., (2018) attributed the pathogenesis of early-onset preeclampsia to the impaired trophoblastic invasion of spiral arteries; which results in placental ischemia and oxidative stress $(21,22)$. Moreover, many studies have reported that uterine artery Doppler ultrasonography is used to measure the high-resistance blood flow in uteroplacental circulation (23). On the other hand, Selvaraj et al., (2016) demonstrated that uterine artery Doppler scan in the first trimester detected only $40 \%$ of the preeclamptic patients, and it showed low positive predictive values for preeclampsia in a low-risk population (24).

Concerning the sensitivity of the placental volume and indices, our results agree with those concluded by Schuchter et al., (2001) who evaluated placental volume and uterine artery Doppler for the prediction of preeclampsia during the first trimester and found that preeclampsia occurred in almost $20.0 \%$ of all pregnancies $(25)$.

Our study revealed that women with preeclampsia had significantly lower placental volume, VI, FI, and VFI than normal ones (P[?] 0.001). This is in agreement with those concluded by Noguchi et al., (2009), who studied a group consisted of 208 normal women with thirteen growth-restricted fetuses. Their results revealed that the majority of preeclamptic women had significantly lower VI, FI, and VFI (26). Similar results were concluded by Rizzo et al., (2009) and Lai et al., (2010) who found statistically significant differences, between normal and preeclamptic women in VI, FI, and VFI. One of the most recent studies conducted by Soliman 
et al., (2019) has revealed high significant statistical differences, between preeclamptic and normal women in both placental VI and VFI $(\mathrm{P}=0.03)$, but on the contrary, no significant statistical difference was observed in placental FI $(3,5,27)$.

In concordance with our findings, Rizzo et al., (2009) reported that placental assessment, including its structural changes and placental volume, can provide better insight into the pathophysiology of preeclampsia, as well as its early prediction (5). Moreover, the study of the placenta can help a better understanding of its pathological and pathophysiological changes with preeclampsia and FGR (28).

In agreement with our findings, Ali et al., (2018) examined 50 pregnant women using 3D-PD ultrasound as a screening tool to assess the uteroplacental circulation in early pregnancy for the prediction of preeclampsia. They concluded that the values of VI, FI, and VFI in preeclamptic patients were significantly lower than in non-preeclamptic women $(\mathrm{P}<0.001)$. Consequently, 3D-PD ultrasound is regarded as a potentially effective screening tool for early prediction of preeclampsia (29). Similarly, Costa et al., (2010) found that VI, FI, and VFI were lower in preeclampsia cases in comparison with normal pregnancy cases in all regions of the placenta. They added that using 3D ultrasound to assess placental vascularity and flow intensity appears to be an interesting research tool (30).

Regarding using a combination of uterine artery indices and placental volume values, Schuchter et al., (2001) evaluated both placental volume and uterine artery Doppler, in the first trimester to predict preeclampsia. They concluded that such combination could help to predict women at risk for development of preeclampsia and other pregnancy complications which was consistent with our results (25).

Furthermore, Hashish et al., (2015) assessed placental volume and vascularization indices at $11^{\text {th }}-13^{\text {th }}$ weeks of gestation and uterine artery Doppler scan (RI and PI) at $21^{\text {st }}-22^{\text {nd }}$ weeks. They concluded that the mean values of placental volume, VI and VFI in the high-risk group were statistically significantly lower $(<0.001,<0.001$ and $<0.002$ respectively). They also found that uterine artery RI and PI were significantly higher in the study group (0.011 and $<0.001$ respectively). In addition, a negative correlation (-0.36) was observed between uterine artery PI and both placental volume and vascularization indices (31). Hafner et al., (2006) observed a strong association between low placental volume in the first trimester and abnormal uterine Doppler in the second trimester. Besides, both the placental volume at 12 weeks of gestation and uterine Doppler screening at 22 weeks showed similar sensitivities for the prediction of preeclampsia (32).

On the other hand, Oznur et al., (2014) examined the effect of placental volume at 11-13 weeks of gestation on the subsequent development of preeclampsia. They found that the placental volume values between the preeclamptic group and the unaffected group were not significantly different. Therefore, placental volume alone is not reliable for the prediction of preeclampsia, but it can be helpful when it is combined with other factors associated with patients (33). Similarly, Hafner et al., (2006) found that placental volume had a low sensitivity for the prediction of preeclampsia (32). Soongsatitanon et al., (2019) concluded that cases with preeclampsia and/or FGR have smaller placental volume. They added that, although the placental volume is not regarded as an effective screening tool for preeclampsia and/or FGR in the first trimester, yet it could be helpful in the prediction of early-onset preeclampsia (34).

In agreement with our findings, it has been reported that the accuracy of prediction of preeclampsia increased when a combination method for detection is used. In other words, uterine artery Doppler alone displays poor accuracy and hence, its combined use with the placental vascular indices was shown to be more accurate in detecting preeclampsia. The development of a reliable accurate screening tool in the first trimester for preeclampsia would enable future research into potential interventions that would potentially lead to improvement in the morbidities and mortalities related to these conditions.

\section{Conclusion}

A statistically significant difference exists between preeclamptic and normotensive pregnant women as regard uterine artery Doppler indices, placental volume and placental vascular indices in the first trimester. Combined screening of uterine artery Doppler with placental volume and vascular indices in the first trimester 
could be beneficial to increase the accuracy of early prediction of preeclampsia and complications of uteroplacental insufficiency.

\section{Declarations}

\section{Availability of data and materials}

Data will be available from the corresponding author upon request.

\section{Conflict of Interest}

The authors declare that they have no competing interests

\section{Funding}

There is no specific grant from any funding agency.

\section{Acknowledgements}

None

\section{Ethics approval and consent to participate}

The Ethics Committee of Faculty of Medicine at El-Minia University approved the study protocol under registration number 1876-5-m-2018, and all subjects gave their informed consent.

\section{Consent for publication}

All authors have given approval to the final version of the manuscript.

\section{Authors' contributions}

Ameer Abdallah, Mohamed Tawfik and Eissa Khalifa designed and supervised the research. Safaa Mohamed, Ameer Abdallah and Eissa Khalifa conducted the study. Mazen Abdel-Rasheed analyzed the data. Sondos Salem and Mazen Abdel-Rasheed wrote the manuscript. All authors have read and approved the manuscript.

\section{References}

1. Jeyabalan A. Epidemiology of preeclampsia: impact of obesity. Nutr Rev. 2013;71(suppl_1):S18-25.

2. Nathan HL, Seed PT, Hezelgrave NL, De Greeff A, Lawley E, Conti-Ramsden F, et al. Maternal and perinatal adverse outcomes in women with pre-eclampsia cared for at facility-level in South Africa: a prospective cohort study. J Glob Health. 2018;8(2).

3. Soliman E, Emarah M, E. Shaheen A, E. Yassin H. Three-dimensional sonographic assessment of placental volume and vascularization in pregnancies complicated by hypertensive disorders. Menoufia Med J. 2019 Oct 1;32(4):1350-4.

4. Baergen RN, Gersell DJ, Kraus FT. Diseases of the Placenta. In: Kurman RJ, Hedrick Ellenson L, Ronnett BM, editors. Blaustein's Pathology of the Female Genital Tract [Internet]. Cham: Springer International Publishing; 2019 [cited 2020 Apr 20]. p. 1223-305. Available from: https://doi.org/10.1007/978-3-31946334-6_19

5. Rizzo G, Capponi A, Pietrolucci ME, Capece A, Arduini D. First-trimester placental volume and vascularization measured by 3-dimensional power Doppler sonography in pregnancies with low serum pregnancyassociated plasma protein a levels. J Ultrasound Med. 2009;28(12):1615-22.

6. Albu A, Anca A, Horhoianu V, Horhoianu I. Predictive factors for intrauterine growth restriction. J Med Life. 2014;7(2):165.

7. Abdallah A, Shawki H, Abdel-Rasheed M, Salem S, Hosni M. Role of 3-D Transvaginal Ultrasonography in Women Undergoing in Vitro Fertilization/Intra-cytoplasmic Sperm Injection. Ultrasound Med Biol. 2020 Jun 1;46(6):1424-7. 
8. Papageorgiou T, Guibert J, Goffinet F, Patrat C, Fulla Y, Janssens Y, et al. Percentile curves of serum estradiol levels during controlled ovarian stimulation in 905 cycles stimulated with recombinant FSH show that high estradiol is not detrimental to IVF outcome. Hum Reprod. 2002;17(11):2846-50.

9. Pedroso MA, Palmer KR, Hodges RJ, da Silva Costa F, Rolnik DL. Uterine artery Doppler in screening for preeclampsia and fetal growth restriction. Rev Bras Ginecol E ObstetriciaRBGO Gynecol Obstet. 2018;40(05):287-93.

10. Papastefanou I, Chrelias C, Siristatidis C, Kappou D, Eleftheriades M, Kassanos D. Placental volume at 11 to 14 gestational weeks in pregnancies complicated with fetal growth restriction and preeclampsia. Prenat Diagn. 2018;38(12):928-35.

11. Duan J, Chabot-Lecoanet A-C, Perdriolle-Galet E, Christov C, Hossu G, Cherifi A, et al. Utero-placental vascularisation in normal and preeclamptic and intra-uterine growth restriction pregnancies: third trimester quantification using 3D power Doppler with comparison to placental vascular morphology (EVUPA): a prospective controlled study. BMJ Open. 2016;6(3):e009909.

12. Salavati N, Smies M, Ganzevoort W, Charles AK, Erwich JJ, Plosch T, et al. The possible role of placental morphometry in the detection of fetal growth restriction. Front Physiol. 2019;9:1884.

13. Tomimatsu T, Mimura K, Matsuzaki S, Endo M, Kumasawa K, Kimura T. Preeclampsia: Maternal Systemic Vascular Disorder Caused by Generalized Endothelial Dysfunction Due to Placental Antiangiogenic Factors. Int J Mol Sci. 2019;20(17):4246.

14. Kristensen JH, Basit S, Wohlfahrt J, Damholt MB, Boyd HA. Pre-eclampsia and risk of later kidney disease: nationwide cohort study. bmj. 2019;365:11516.

15. Yucesoy G, Ozkan S, Bodur H, Tan T, Calışkan E, Vural B, et al. Maternal and perinatal outcome in pregnancies complicated with hypertensive disorder of pregnancy: a seven year experience of a tertiary care center. Arch Gynecol Obstet. 2005;273(1):43-9.

16. Musa J, Mohammed C, Ocheke A, Kahansim M, Pam V, Daru P. Incidence and risk factors for preeclampsia in Jos Nigeria. Afr Health Sci. 2018;18(3):584-95.

17. Zhong Y, Tuuli M, Odibo AO. First-trimester assessment of placenta function and the prediction of preeclampsia and intrauterine growth restriction. Prenat Diagn Publ Affil Int Soc Prenat Diagn. 2010;30(4):293308.

18. Bujold E, Morency A-M, Roberge S, Lacasse Y, Forest J-C, Giguère Y. Acetylsalicylic acid for the prevention of preeclampsia and intra-uterine growth restriction in women with abnormal uterine artery Doppler: a systematic review and meta-analysis. J Obstet Gynaecol Can. 2009;31(9):818-26.

19. Townsend R, O'Brien P, Khalil A. Current best practice in the management of hypertensive disorders in pregnancy. Integr Blood Press Control. 2016;9:79.

20. Myatt L, Clifton RG, Roberts JM, Spong CY, Hauth JC, Varner MW, et al. The utility of uterine artery Doppler velocimetry in prediction of preeclampsia in a low-risk population. Obstet Gynecol. 2012;120(4):815.

21. Visser GH, Bilardo CM, Lees C. Fetal growth restriction at the limits of viability. Fetal Diagn Ther. 2014;36(2):162-5.

22. Schoots MH, Gordijn SJ, Scherjon SA, van Goor H, Hillebrands J-L. Oxidative stress in placental pathology. Placenta. 2018;69:153-61.

23. García B, Llurba E, Valle L, Gómez-Roig M, Juan M, Perez-Matos C, et al. Do knowledge of uterine artery resistance in the second trimester and targeted surveillance improve maternal and perinatal outcome? UTOPIA study: a randomized controlled trial. Ultrasound Obstet Gynecol. 2016;47(6):680-9. 
24. Selvaraj LR, Rose N, Ramachandran M. First trimester screening for pre-eclampsia and fetal growth restriction. J Fetal Med. 2016;3(2):77-84.

25. Schuchter K, Metzenbauer M, Hafner E, Philipp K. Uterine artery Doppler and placental volume in the first trimester in the prediction of pregnancy complications. Ultrasound Obstet Gynecol Off J Int Soc Ultrasound Obstet Gynecol. 2001;18(6):590-2.

26. Noguchi J, Hata K, Tanaka H, Hata T. Placental vascular sonobiopsy using three-dimensional power Doppler ultrasound in normal and growth restricted fetuses. Placenta. 2009;30(5):391-7.

27. Lai P, Wang Y, Welsh A. Reproducibility of regional placental vascularity/perfusion measurement using 3D power Doppler. Ultrasound Obstet Gynecol. 2010;36(2):202-9.

28. Roberts JM, Escudero C. The placenta in preeclampsia. Pregnancy Hypertens Int J Womens Cardiovasc Health. 2012;2(2):72-83.

29. Ali SA, Mansour MGE-D, Farouk O, Mohammed WE. Efficiency of placental three dimentional power Doppler ultrasonography for predicting preeclampsia in early pregnancy. Egypt J Radiol Nucl Med. 2018;49(3):8928.

30. Costa J, Rice H, Cardwell C, Hunter A, Ong S. An assessment of vascularity and flow intensity of the placenta in normal pregnancy and pre-eclampsia using three-dimensional ultrasound. J Matern Fetal Neonatal Med. 2010;23(8):894-9.

31. Hashish N, Hassan A, El-Semary A, Gohar R, Youssef M. Could 3D placental volume and perfusion indices measured at 11-14 weeks predict occurrence of preeclampsia in high-risk pregnant women? J Matern Fetal Neonatal Med. 2015;28(9):1094-8.

32. Hafner E, Metzenbauer M, Hofinger D, Stonek F, Schuchter K, Waldhor T, et al. Comparison between three-dimensional placental volume at 12 weeks and uterine artery impedance/notching at 22 weeks in screening for pregnancy-induced hypertension, pre-eclampsia and fetal growth restriction in a low-risk population. Ultrasound Obstet Gynecol Off J Int Soc Ultrasound Obstet Gynecol. 2006;27(6):652-7.

33. Dundar O, Olgac Y, Acar D, Ekiz A, Yıldırım G, Gedikbaşı A. The role of measuring PAPP-A and placental volume for the prediction of preeclampsia at 11-14 weeks of gestation. Perinat JournalPerinatoloji Derg. 2014;22(1).

34. Soongsatitanon A, Phupong V. First trimester 3D ultrasound placental volume for predicting preeclampsia and/or intrauterine growth restriction. J Obstet Gynaecol. 2019;39(4):474-9.

Table (1): Difference between the preeclampsia and normotensive group

\begin{tabular}{llll}
\hline & $\begin{array}{l}\text { Preeclampsia group } \\
(\mathbf{n = 1 6 3})\end{array}$ & $\begin{array}{l}\text { Normotensive group } \\
(\mathbf{n = 1 8 5 6})\end{array}$ & P-value \\
\hline Age (year) & $24.75 \pm 5.85$ & $24.53 \pm 5.78$ & 0.646 \\
BMI $\left(\mathrm{kg} / \mathrm{m}^{2}\right)$ & $25.4 \pm 1.93$ & $25.13 \pm 2.29$ & 0.088 \\
$\begin{array}{l}\text { Parity Nulliparous } \\
\text { Multiparous }\end{array}$ & $97(59.5 \%) 66(40.5 \%)$ & $897(48.3 \%) 959(51.7 \%)$ & $0.007^{*}$ \\
$\begin{array}{l}\text { Gestational age at } \\
\text { delivery (weeks) }\end{array}$ & $35.2 \pm 0.67$ & $36.67 \pm 1.57$ & $0.026^{*}$ \\
$\begin{array}{l}\text { Previous History of } \\
\begin{array}{l}\text { Preeclampsia } \\
\text { Family History of }\end{array}\end{array}$ & $158 / 163(96.9 \%)$ & $370 / 1856(19.9 \%)$ & $<0.001^{*}$ \\
$\begin{array}{l}\text { Preeclampsia } \\
\text { Placental volume (ml) }\end{array}$ & $109 / 163(66.9 \%)$ & $62 / 1856(3.3 \%)$ & $<0.001^{*}$ \\
Placental VI & $77.09 \pm 8.1$ & & \\
& $7.41 \pm 0.69$ & $99.83 \pm 9.83$ & $<0.001^{*}$ \\
& & $9.89 \pm 1.5$ & $<0.001^{*}$
\end{tabular}




\begin{tabular}{llll}
\hline & $\begin{array}{l}\text { Preeclampsia group } \\
(\mathbf{n = 1 6 3 )}\end{array}$ & $\begin{array}{l}\text { Normotensive group } \\
(\mathbf{n = 1 8 5 6 )}\end{array}$ & P-value \\
\hline Placental FI & $39.03 \pm 4.43$ & $46.63 \pm 4.53$ & $<0.001^{*}$ \\
Placental VFI & $2.77 \pm 0.58$ & $4.4 \pm 0.92$ & $<0.001^{*}$ \\
Mean Uterine PI & $2.02 \pm 0.48$ & $1.11 \pm 0.26$ & $<0.001^{*}$ \\
Mean Uterine RI & $0.83 \pm 0.09$ & $0.64 \pm 0.11$ & $<0.001^{*}$ \\
$\begin{array}{l}\text { Development of FGR } \\
\text { Neonatal birth weight }\end{array}$ & $67 / 160(41.9 \%)^{*}$ & $247 / 1856(13.3 \%)$ & $<0.001^{*}$ \\
(gram) & $2252.22 \pm 235.44^{\#}$ & $2655.95 \pm 187.33$ & $<0.001^{*}$ \\
Neonatal complications & $105 / 160(65.6 \%)^{*}$ & $710 / 1856(38.3 \%)$ & $<0.001^{*}$ \\
NICU admission & $74 / 160(46.3 \%)^{*}$ & $278 / 1856(15 \%)$ & $<0.001^{*}$ \\
\hline
\end{tabular}

VI: vascularization Index, FI: flow index, VFI: vascularization flow index, PI: pulsatility index, RI: resistance index, FGR: fetal growth retardation, NICU: neonatal intensive care unit; Normally distributed variables are represented as mean $\pm \mathrm{SD}$ and Unpaired Student's t-test was used; Categorical variables are represented as number (\%) and Pearson Chi-square test was used; *: Statistically significant (p-value $<0.05$ ); : 3 cases are not included as IUFD developed

Table (2): Performance indices for the first-trimester 3D ultrasound indices for prediction of preeclampsia

\begin{tabular}{llllllllll}
\hline & Cut off Value & Sensitivity & Specificity & LR + & LR- & AUC & P-value & $95 \%$ CI & 95\% CI \\
\hline & & & & & & & & Lower & Upper \\
Placental Volume & 86.955 & $89.0 \%$ & $93.2 \%$ & 13.00 & 0.12 & 0.970 & $<0.001^{*}$ & 0.962 & 0.979 \\
Placental VI & 8.545 & $97.5 \%$ & $82.1 \%$ & 5.45 & 0.03 & 0.964 & $<0.001^{*}$ & 0.954 & 0.973 \\
Placental FI & 42.255 & $76.1 \%$ & $80.9 \%$ & 3.98 & 0.30 & 0.876 & $<0.001^{*}$ & 0.848 & 0.903 \\
Placental VFI & 3.755 & $98.8 \%$ & $70.3 \%$ & 3.32 & 0.02 & 0.934 & $<0.001^{*}$ & 0.919 & 0.950 \\
Mean Uterine PI & 1.485 & $87.1 \%$ & $96.3 \%$ & 23.78 & 0.13 & 0.972 & $<0.001^{*}$ & 0.963 & 0.982 \\
Mean Uterine RI & 0.785 & $71.2 \%$ & $92.2 \%$ & 9.11 & 0.31 & 0.909 & $<0.001^{*}$ & 0.889 & 0.930 \\
\hline
\end{tabular}

VI: vascularization Index, FI: flow index, VFI: vascularization flow index, PI: pulsatility index, RI: resistance index, LR: likelihood ratio, AUC: area under the curve, CI: confidence interval; *: Statistically significant (p-value $<0.05)$

Table (3): Regression model for prediction of preeclampsia using clinical and 3D ultrasound criteria for utero- placental blood flow

\begin{tabular}{lllll}
\hline & Univariate regression & Univariate regression & Multivariate regression & Multivariate regressi \\
\hline & Crude OR $(95 \% \mathrm{CI})$ & $\mathrm{P}$ value & Adjusted OR $(95 \% \mathrm{CI})$ & $\mathrm{P}$ value \\
Placental Volume & $0.764(0.738-0.791)$ & $<0.001^{*}$ & $0.899(0.83-0.974)$ & $0.009^{*}$ \\
Placental VI & $0.053(0.035-0.081)$ & $<0.001^{*}$ & $0.295(0.143-0.609)$ & $<0.001^{*}$ \\
Placental FI & $0.689(0.655-0.724)$ & $<0.001^{*}$ & $0.97(0.867-1.087)$ & 0.603 \\
Placental VFI & $0.038(0.023-0.061)$ & $<0.001^{*}$ & $0.597(0.279-1.281)$ & 0.186 \\
Mean Uterine PI & $4.536(1.539-13.363)$ & $0.006^{*}$ & $7.12(2.186-23.185)$ & $<0.001^{*}$ \\
Mean Uterine RI & $87.435(1.946-3929.466)$ & $0.021^{*}$ & $2.564(0.009-744.293)$ & 0.745 \\
Past history of PE & $156(19.425-1252.816)$ & $<0.001^{*}$ & $98.948(23.081-424.193)$ & $<0.001^{*}$ \\
Family history of PE & $60.231(12.693-285.813)$ & $<0.001^{*}$ & $13.883(3.656-52.727)$ & $<0.001^{*}$ \\
\hline
\end{tabular}

VI: vascularization Index, FI: flow index, VFI: vascularization flow index, PI: pulsatility index, RI: resistance 
index, PE: preeclampsia, OR: odd ratio, CI: confidence interval; *: Statistically significant (p-value $<0.05)$

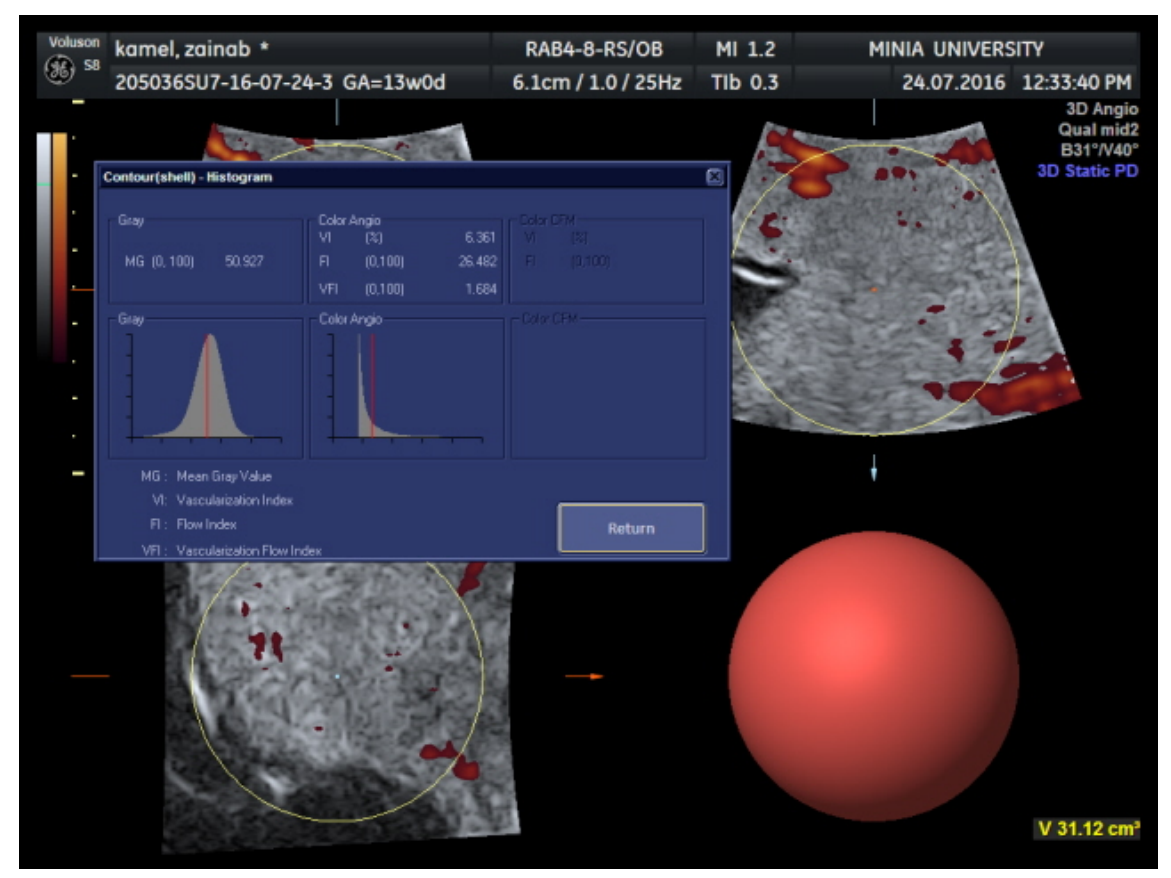

Figure 1: Placental volume and vascularization of a case of normal pregnancy during the first trimester.

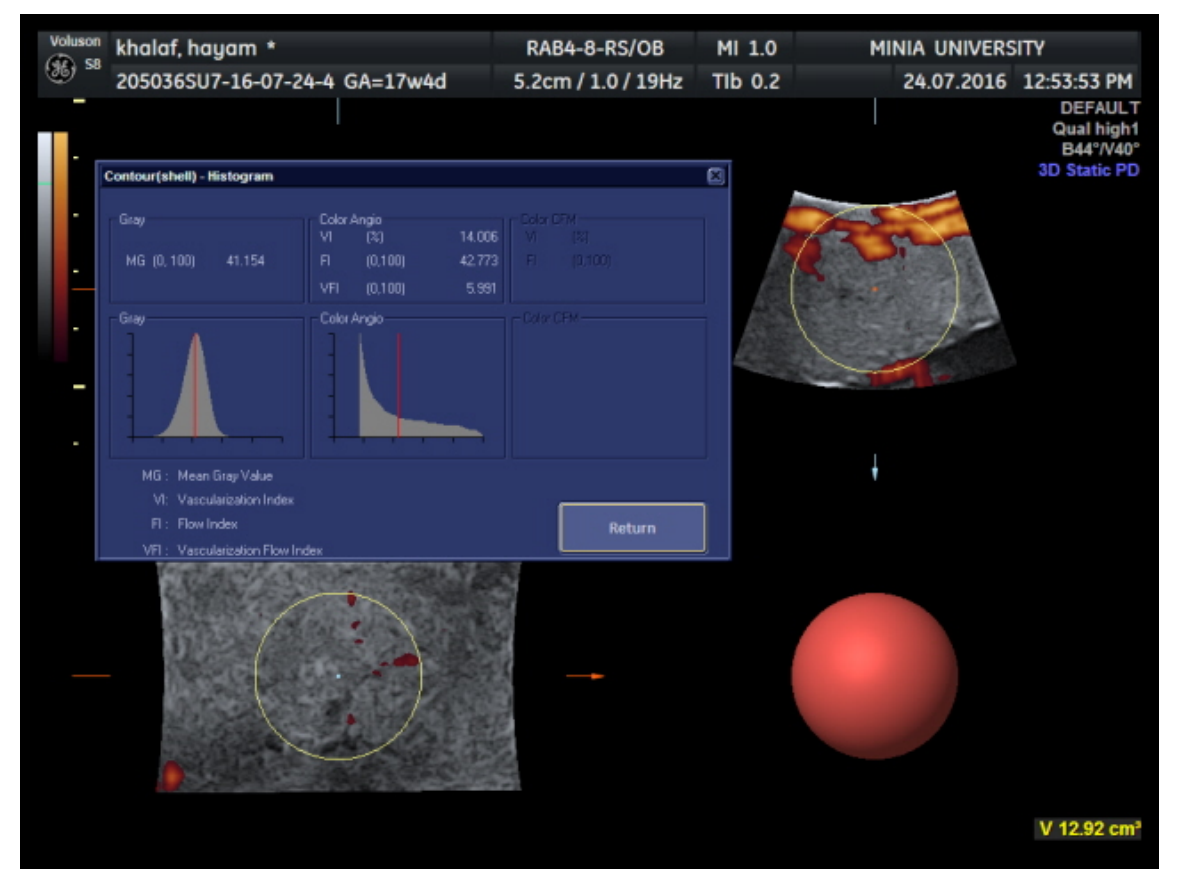

Figure 2: Placental volume and vascularization of a case of preeclampsia during the first trimester. 


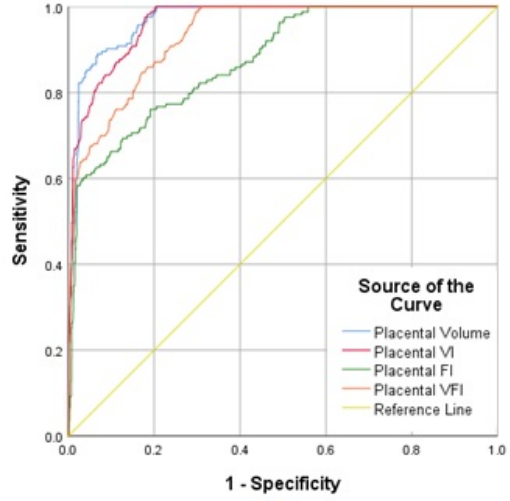

A

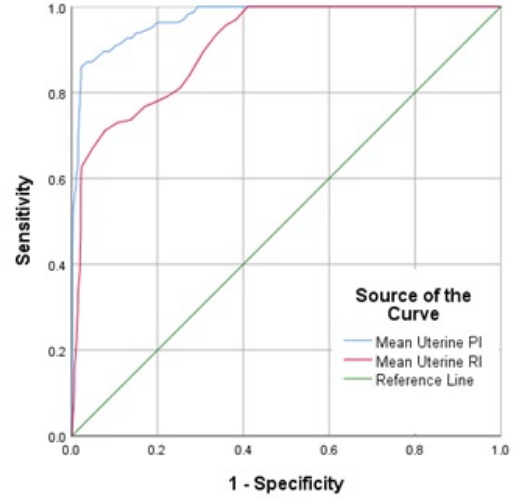

B

Figure 3: Roc curve analysis of the placental indices (A) and the uterine artery indices (B) 